\title{
Calidad de las tesis en la escuela universitaria de enfermería en Lima-Perú, período 2011-2015
}

\author{
Diego Armando Guzmán Díaz, ${ }^{1, a}$
}

Guzmán DDA. Calidad de las tesis en la escuela universitaria de enfermería en Lima-Perú, período 20112015. Cuid salud, ene-jun 2017; 3(1):288-295.

\section{RESUMEN}

Introducción: la elaboración de tesis de enfermería, la redacción lógica y su poca divulgación pone en interrogante su calidad en las escuelas universitarias de enfermería, especialmente porque los organismos nacionales e internacionales reconocen que esta producción es una función principal de la Universidad, debiendo cumplirse con el último paso del proceso investigativo que es la publicación. Objetivo: determinar la calidad de las tesis producidas en la Escuela de Enfermería Padre Luis Tezza (EEPLT) durante el periodo 2011-2015. Material y método: estudio de enfoque cuantitativo, tipo aplicado y diseño exploratorio descriptivo, cuya población de estudio fue de 132 tesis registradas en la Biblioteca Institucional. El instrumento fue una ficha de registro, el cual fue sometido a validez de juicio de expertos; recolectándose los datos en diciembre 2016; utilizando para su análisis la estadística descriptiva en programa Excel 2013. Resultados: las tesis de la EEPLT fueron de buena $(36 \%)$ y regular calidad (36\%); apenas el $8 \%$ fue divulgado en revistas científicas y $23 \%$ citado en otras investigaciones. Conclusiones: las tesis del periodo 2011-2015 en la EEPLT fueron calificadas de buena y regular calidad. Siendo necesario continuar trabajando en esta área de formación universitaria fortaleciendo la divulgación e impacto de la producción científica.

Palabras clave: calidad, investigación, enfermería, divulgación, tesis académica (Fuente: DeCS BIREME).
Guzmán DDA. Quality of theses in a university nursing school in Lima-Peru, period 2011-2015. Cuid salud, enejun 2017; 3(1):288-295.

\begin{abstract}
Introduction: The development of nursing theses, the logical wording and its lack of divulgation raises questions about their quality in university nursing schools, especially because national and international organizations recognize that this production is a main function of the University, and must be fulfilled the last step of the investigative process that is the publication.

Objective: To determine the quality of the theses produced in the School of Nursing Father Luis Tezza (EEPLT) during the period 2011-2015. Material and method: Study of quantitative approach, applied type and descriptive exploratory design, whose study population was 132 theses registered in the Institutional library. The instrument was a registration form, which was subject to the validity of expert judgment; the data being collected in December 2016; using descriptive statistics in the Excel 2013 program. Results: The theses of the EEPLT were good (36\%) and regular quality $(36 \%)$; only $8 \%$ was reported in scientific journals and $23 \%$ cited in other research. Conclusions: The theses of the period 2011-2015 in the EEPLT were described as good and regular quality. It is necessary to continue working in this area of university education by strengthening the dissemination and impact of scientific production.
\end{abstract}

Keywords: Quality, research, nursing, outreach, academic dissertations (Source: MeSH NLM)

1 Hospital Nacional Hipólito Unanue. Lima-Perú.

a Licenciado en Enfermería. 


\section{INTRODUCCIÓN}

El ser humano siempre tuvo un concepto intuitivo de la calidad, en razón de la búsqueda y el afán de perfeccionamiento, como constantes de sus obras a través de la historia. La raíz etimológica de la palabra calidad está en el término griego Kalos que significa lo bueno, lo apto, y también en la palabra latín qualitem, que significa cualidad o propiedad. ${ }^{1}$

El Sistema Nacional de Evaluación, Acreditación y Certificación de la Calidad Educativa (SINEACE) ${ }^{2}$, citando a Harvey y Knight, refiere que uno de los cinco significados atribuidos a la calidad es la transformación, definiéndose a la calidad como un proceso de cambio cualitativo que enfatiza otorgar valor y empoderar a los estudiantes.

Según Nicholson, ${ }^{3}$ en la actualidad, la definición internacional más aceptada sobre calidad en la educación superior es su capacidad (fitness) para cumplir con el propósito de cada institución educativa. El atractivo de esta definición radica en su flexibilidad inherente, que permite a las instituciones medir su calidad en términos de su capacidad para cumplir sus respectivas misiones y objetivos.

La Ley General de Educación del Perú del 2003, en su artículo $29^{\circ}$ refiere que el sistema educativo comprende la educación básica y superior. La segunda destinada ineludiblemente a la investigación, creación y difusión de conocimientos; además de su proyección a la comunidad y al logro de competencias profesionales de alto nivel, acorde con la demanda y la necesidad del desarrollo sostenible de cada país. Aunque en los últimos años se han entregado títulos universitarios sin cumplir con el producto científico.

La Organización de las Naciones Unidas para la Educación, la Ciencia y la Cultura-UNESCO ${ }^{5}$ reconoce que la educación superior es una responsabilidad pública y su cuota al desarrollo sostenible de las naciones solo será posible en la medida que las instituciones formen profesionales con pensamiento crítico y autónomos en el campo de la investigación y la innovación, cabiéndole promover el desarrollo del capital humano, las tecnologías de información y comunicación, la transferencia de conocimientos y la resolución de problemas en cada sociedad. Al respecto, en Enfermería, como disciplina que ejecuta preferentemente investigación aplicada y cumpliendo con las premisas de la UNESCO, la investigación debería validar y mejorar el conocimiento existente y generar otros nuevos que influyan en su ejercicio profesional.
Así siendo, la productividad investigativa es un indicador esencial en toda institución de educación superior, sobre todo, porque se relaciona con su calidad y porque a través de ella se evalúan el logro de sus metas; identificando fortalezas y debilidades en el sistema de investigación. ${ }^{6}$ Al respecto, Paravic y Burgos $^{7}$ refieren que la evaluación de la producción científica constituye un elemento clave en la toma de decisiones que propicia la mejora de la institución o del programa evaluado, contribuye a su transformación y promueve espacios de reflexión y profundización teórica en el área de investigación; además de su control de calidad.

Los mismos autores, citando a Rosas, afirman que las evaluaciones periódicas de programas, tesis y autores no son frecuentes en las universidades y facultades/escuelas, quedando oculto las deficiencias e imposibilitando su retroalimentación. ${ }^{7}$ Hecho que pudo corroborarse en el estudio de Merighi et al.; ${ }^{8}$ a nivel nacional, las tesis en su mayoría se califican como no aceptables $^{9,10}$ o de regular calidad. ${ }^{11}$

En concordancia con la nueva Ley Universitaria, ${ }^{12}$ la EEPLT enfatiza en su misión la investigación y hasta diciembre del 2016 había producido 427 en sus 33 años de fundación. Siendo que desde el 2010 cuenta con diversos reglamentos que norman el desarrollo de la investigación $^{13}$ y, entre el 2009-2016 se sustentaron el $42 \%$ de tesis. En tal sentido, para evaluar su desempeño en la investigación este estudio tiene como objetivo determinar el nivel de calidad de las tesis producidas por la EEPLT en el periodo 2011-2015, además de conocer la línea de investigación en la que se encuadran, el porcentaje de tesis que incluyen en el marco teórico referencial una teoría de enfermería.

\section{MATERIAL Y MÉTODOS}

El estudio fue de enfoque cuantitativo, de tipo aplicado, método no experimental, diseño exploratorio descriptivo.

El área de estudio fue la EEPLT afiliada a la Universidad Ricardo Palma-Lima; dicha institución ofrece educación superior de nivel universitario y congrega generalmente a estudiantes que pertenecen a los estratos socioeconómicos C y D, en general, provenientes de todo el país, habiendo sido acreditada por SINEACE el 2013. ${ }^{14}$

La población universo fue constituida por 135 tesis sustentadas entre 2011-2015 constituyendo el 100\%. De ese total, tres tesis (uno de posgrado y dos de pregrado) se encuadraron dentro de los criterios de eliminación 
quedando $132(97,7 \%)$ que conformaron la población de estudio.

Para la recolección de datos se usó la técnica de análisis documental utilizando una Ficha de Registro, elaborado a partir de estudio previo. ${ }^{11}$ La Ficha se compone de tres grandes componentes; Datos Generales (codificación, año de sustentación, línea de investigación y teoría de enfermería empleada), Evaluación de las tesis y Leyenda (instructivo de evaluación). El componente Evaluación comprende todos los aspectos de cualificación de la calidad y está conformado por 4 dimensiones: 1) proceso de investigación y rigor del método científico (19 ítems); 2) redacción y orden lógico del tema a investigar (4 ítems); 3) divulgación (3 ítems) y, 4) impacto (1 ítem).

Las puntuaciones de las respuestas oscilaron entre cero (0), 1 y 2 puntos, siendo el máximo puntaje 54 y mínimo cero (0). Los puntajes finales fueron traducidos al sistema vigesimal considerando una escala métrica: Muy malo de 0- 5,99; Malo de 6-10,99; Regular de 1113,99; Bueno de 14-15,99; Muy bueno de 16-18,99 y Excelente de 19-20. ${ }^{15}$

La Ficha fue sometida a juicio de expertos constituido por ocho especialistas en investigación, quienes sugirieron mejorar la calificación respecto a los estudios descriptivos y tipos de muestreo. Dichos resultados se analizaron con la prueba binomial $(p=0,0235)$. Culminada esta fase fue obviada la confiabilidad por la naturaleza del instrumento.

Previo a la recolección de datos se cumplieron con los procedimientos administrativos; luego se contactó con la responsable de la biblioteca de la EEPLT. Los datos fueron recolectados por el investigador en el mes de diciembre 2016, revisando las tesis en formato físico y con ayuda de un ordenador se verificaba la información en línea (dimensión 3 y 4).

En las dimensiones "divulgación", "difusión" e "impacto" se utilizó inicialmente los archivos de los eventos científicos organizados por la EEPLT (20092015). Luego, se iniciaba la búsqueda a través de internet con el nombre del autor, título de la tesis y/o palabras clave en revistas electrónicas nacionales y por último, se hacía uso de las bases de datos mediante el buscador google.scholar o directamente en Scielo, Redalyc. Además, se empleó el Registro Nacional de Trabajos de Investigación (RETANI), Cybertesis URP, Consejo Nacional de Ciencia, Tecnología e Innovación (Concytec), la Biblioteca Virtual de Salud (BVS).
Los datos fueron ingresados a una base de datos creada en el programa Excel 2013 utilizando códigos, luego analizados estadísticamente en el mismo paquete usando la estadística descriptiva (frecuencia, porcentajes).

El proyecto fue sometido a evaluación y aprobado por el Comité de Ética en Investigación de la EEPLT (carta $\mathrm{N}^{\circ}$ 0138-2016/D-EEPLT). La investigación cumplió con utilizar los principios éticos de la información reservándose la confidencialidad de la información, tratándose de un estudio que trabajó con fuente de nivel secundario.

\section{RESULTADOS}

De las 135 tesis, uno era de posgrado y dos no fueron localizadas. Asimismo, se destaca que en el 2014 la autoría de las tesis era por pares, hecho que disminuyó en ese año la cantidad de tesis producidas, en relación con los años previos y posteriores. Seguidamente se presentan las tablas y gráficos del análisis.

Gráfico1: Inclusión de una teoría de enfermería en las tesis del quinquenio 2011-2015. EEPLT-Lima, diciembre 2016.

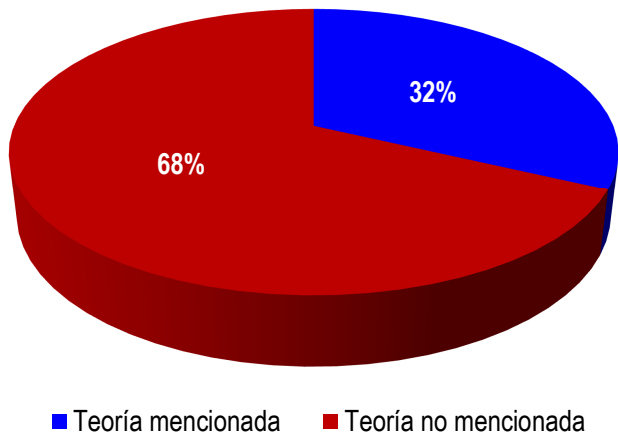

Tabla 1: Adecuación de las tesis del quinquenio 20112015 a las líneas de investigación. EEPLT-Lima, diciembre 2016.

\begin{tabular}{|c|c|c|c|c|c|}
\hline & 2011 & 2012 & 2013 & 2014 & 2015 \\
\hline $\begin{array}{l}\text { Gestión del cuidado de } \\
\text { Enfermería }\end{array}$ & $20 \%$ & $12 \%$ & $0 \%$ & $4 \%$ & $0 \%$ \\
\hline Salud y género & $0 \%$ & $0 \%$ & $9 \%$ & $4 \%$ & $0 \%$ \\
\hline $\begin{array}{l}\text { Promoción y desarrollo } \\
\text { de la salud del niño y } \\
\text { del adolescente }\end{array}$ & $40 \%$ & $47 \%$ & $66 \%$ & $56 \%$ & $78 \%$ \\
\hline $\begin{array}{l}\text { Medio ambiente y salud } \\
\text { del adulto y adulto } \\
\text { mayor }\end{array}$ & $10 \%$ & $21 \%$ & $9 \%$ & $24 \%$ & $6 \%$ \\
\hline Calidad de vida & $25 \%$ & $21 \%$ & $14 \%$ & $8 \%$ & $11 \%$ \\
\hline $\begin{array}{l}\text { Cuidado y educación de } \\
\text { Enfermería en siglo XXI }\end{array}$ & $5 \%$ & $0 \%$ & $3 \%$ & $4 \%$ & $6 \%$ \\
\hline
\end{tabular}


Gráfico 2: Calidad de las tesis en el quinquenio 20112015. EEPLT-Lima, diciembre 2016.

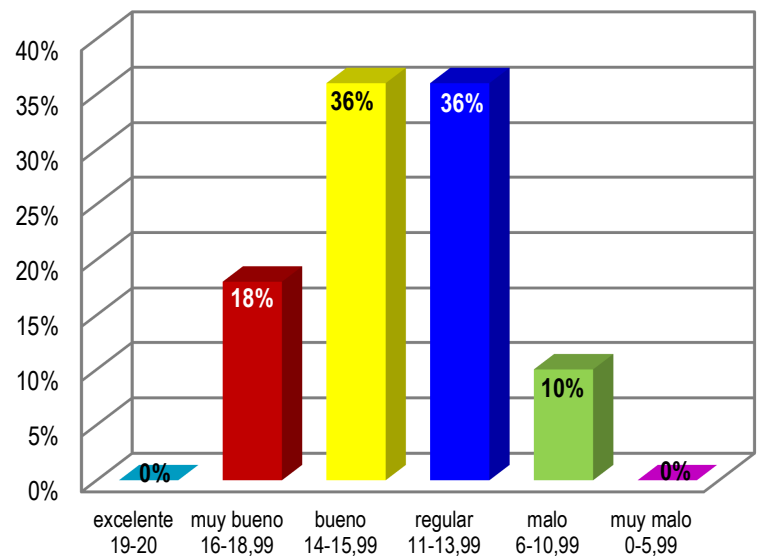

Gráfico 3: Calidad de las tesis en el quinquenio 20112015, según dimensión proceso de investigación y rigor del método científico. EEPLT-Lima, diciembre 2016.

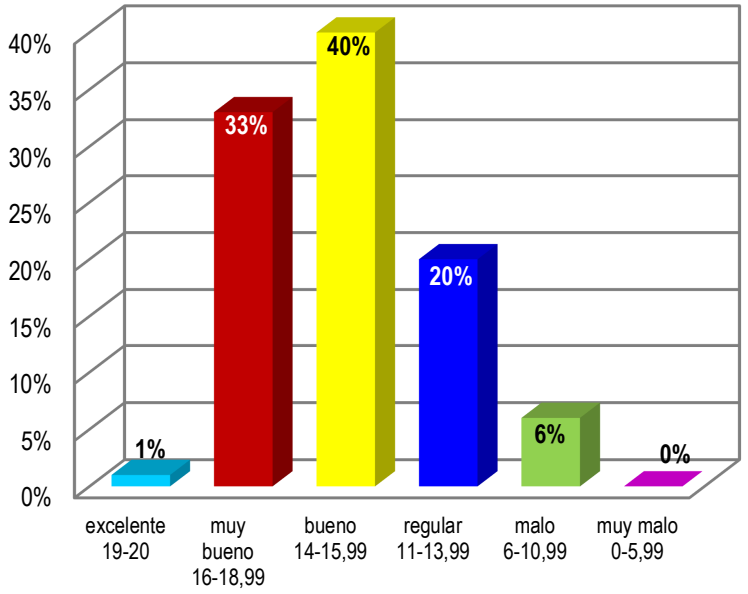

Gráfico 4: Calidad de las tesis en el quinquenio 20112015, según dimensión redacción y orden lógico del tema investigado. EEPLT-Lima, diciembre 2016.

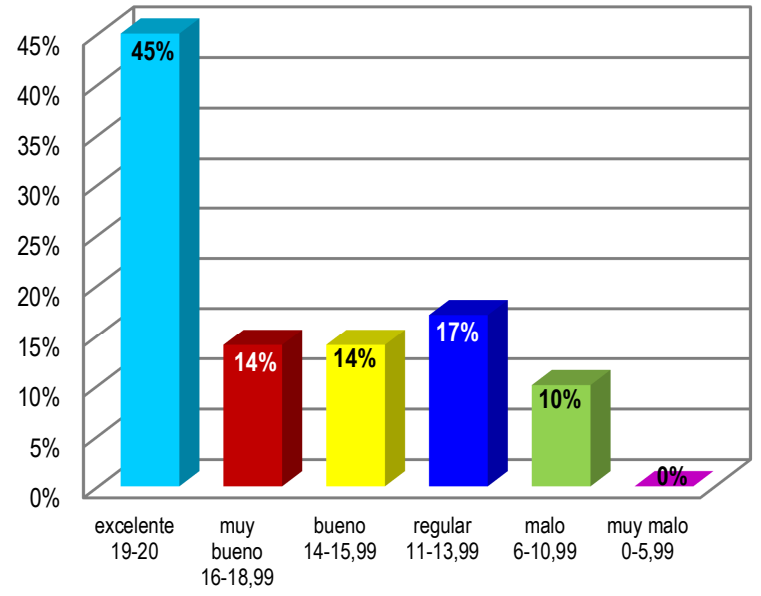

Gráfico 5: Calidad de las tesis en el quinquenio 20112015, según dimensión divulgación. EEPLT-Lima, diciembre 2016.

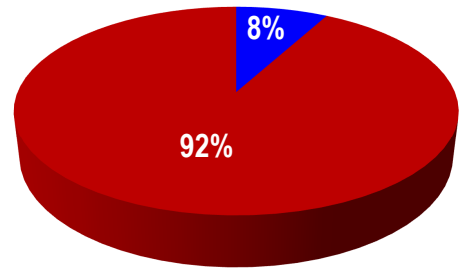

- Tesis publicadas - Tesis no publicadas

Gráfico 6: Calidad de las tesis en el quinquenio 20112015, según dimensión impacto. EEPLT-Lima, diciembre 2016.

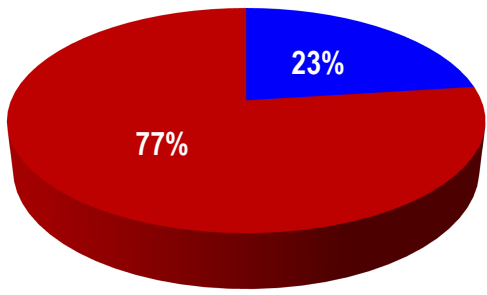

- Tesis mencionada $\quad$ Tesis no mencionada

\section{DISCUSIÓN}

Analizar la calidad educativa en investigación contribuirá a que los futuros profesionales sean más competentes, analíticos y capaces. ${ }^{14}$ Resaltando que es parte de la consciencia universitaria desarrollar nuevas habilidades técnicas, destrezas y conocimiento en bien de la profesión y sociedad; además que es obligación del Estado implementar mecanismos para mantener a la comunidad científica acorde con las exigencias del mundo globalizado en aras de brindar un cuidado de calidad. $^{5}$

Los resultados del estudio muestran que las tesis del quinquenio 2011-2015 comprenden el 31\% del total producido en la EEPLT, lo que significa que la institución cada vez, está apostando más por mejorar el área de investigación. ${ }^{13,14,16}$

La UNESCO ${ }^{5}$ expresa que es función esencial de la universidad generar nuevo conocimiento. En ese sentido, el Estado Peruano impele a los universitarios a concluir su carrera con un aporte al desarrollo de la ciencia y la tecnología en el país. ${ }^{12}$ Responsabilidad que viene siendo supervisada y monitorizada por la SUNEDU, a través de su ente regulador el SINEACE, haciendo énfasis en los estándares del área de investigación en estos últimos años. ${ }^{2}$ 
En el gráfico 1, solo un tercio de las tesis mencionó una teoría de enfermería, componente obligatorio en las tesis de la EEPLT desde el 2013, en aras de comenzar a interpretar los datos obtenidos en las investigaciones bajo un referente teórico y cómo éste ayuda y facilita la comprensión de la naturaleza del cuidado. ${ }^{17}$ Este análisis evaluó la construcción de la teoría de enfermería en el marco teórico referencial adecuado a la problemática en estudio; aunque dichas teorías no siempre son explotadas apropiadamente en el análisis de los resultados, posiblemente por la poca familiaridad de su dominio en la práctica en el contexto peruano. Ciertamente, trasladar la teoría de lo abstracto a lo práctico no es tarea fácil; sería necesario tener un alto dominio de la teoría y de sus componentes para visualizarlos en la práctica y así poder conjugarlos en la cotidianeidad del cuidado.

Pero esta situación no solo se identifica en las tesis de pregrado, también se evidencia en muchas tesis de posgrado. Demostrando que se debe seguir trabajando en el claustro universitario la introducción a la práctica de las teorías y modelos de enfermería, especialmente mediante estudio de casos, discusiones clínicas e investigaciones conjuntas docentes-discentes, tratando de entender cómo las teorías soportan el cuidado al usuario; primero, estudiándolas profundamente para lograr comprenderlas y así poder operativizarlas en situaciones supuestas, y luego, aplicándolas al contexto real con los usuarios, tal y como viene ocurriendo en otros países, como Colombia, Chile, Brasil, dónde las escuelas y facultades asumieron una teoría de enfermería como referente en la enseñanza-aprendizaje $\mathrm{y}$, por supuesto en el cuidado al usuario.

En las líneas de investigación (gráfico 2), destaca la salud del niño y adolescente. Una posible interpretación sería el contacto de las estudiantes con las disciplinas de pediatría y materno infantil en el séptimo ciclo, etapa en que comienzan a elaborar el proyecto de tesis. Si bien el área de materno-infantil y adolescente es prioritario en las políticas sanitarias del país y del mundo, ${ }^{18}$ sería importante también atender las otras demandas sanitarias, como las enfermedades crónicodegenerativas, sobre todo, en el nivel de atención primaria entendiendo las proyecciones en la población al 2030 y sus nefastas consecuencias económicas y sociales.

Juarez-Rolando $^{19}$ refiere que el análisis bibliométrico permite caracterizar el desarrollo de una disciplina y sus líneas de investigación. Pero también muestra los caminos seguidos y hacia dónde se debe ir, en respuesta principalmente a las necesidades sociosanitarias de cada país. Siendo así, los estudios deberían enfatizar aspectos del cuidado directamente relacionados con la ciencia de enfermería, aportando bases referenciales que fundamenten científicamente las acciones de la práctica y aporten en el mejor cuidado a la población y, posiblemente, modificando o incluyendo nuevas líneas de investigación.

Respecto a la calidad de las tesis, la mayor proporción fue calificada entre "buena" y "regular", con un significativo porcentaje de tesis "malas" (grafico 3). Información que merece ser considerada para continuar implementando medidas de mejora en la EEPLT. Ochoa-Vigo et al. $^{13}$ identificaron que las tesis en la EEPLT eran en general de buena calidad; sin embargo, este estudio consideró criterios más rigurosos acorde con las exigencias de altos patrones de producción científica. $^{20}$

Análisis científico de las tesis en el contexto peruano muestran resultados diversos. Por un lado, Pisconte $^{21}$ concluye que las investigaciones de enfermería en la Universidad San Luis Gonzaga eran aceptables; por otro lado, en la Universidad Nacional Santiago Antúnez de Mayolo fue identificado que el $41 \%$ de tesis era poco adecuado. ${ }^{22}$ Frente a eso, vale destacar que el proceso de mejora continua implementado en la EEPLT sobre el desarrollo de la producción científica es favorable con relación a otras universidades. $^{14}$

En la dimensión proceso de investigación y rigor del método científico (grafico 4) se observa una tendencia de buena (40\%) a muy buena calidad $(33 \%)$ en las tesis; previo estudio en rigor metodológico logró calificar como "muy buenas" al 53\% de ellas. ${ }^{21}$ Mientras tanto, cabe admitir que desarrollar una investigación es un acto concienzudo que implica organización, planificación y persistencia ${ }^{23}$ y mucha lectura y relectura crítica reflexiva, a fin de saber elaborar el discurso lógico de las ideas y de los hechos en el informe final.

En el presente estudio, el $45 \%$ de tesis tenía un orden lógico en la secuencia de la descripción del problema de investigación hasta las conclusiones, mientras que un $10 \%$ tenía inadecuación en la ilación del tema (gráfico 5). Siendo importante que la investigación sea clara, sencilla y coherente desde el principio, es decir, del planteamiento del problema a la sección de la discusión, facilitando su lectura a los lectores ${ }^{20}$ y comunidad académica científica.

La divulgación y difusión de la investigación juega un papel fundamental en la forma de cómo el público en general y los interesados, propiamente de la disciplina, 
puedan obtener y confrontar los resultados. ${ }^{13,24}$ El porcentaje de tesis publicadas en revistas científicas nacional y regional alcanzó solo el 8\% (gráfico 6).

Entendiendo que todo conocimiento que no es divulgado no cumple con el último paso de la investigación; $;{ }^{7,20}$ es preocupante que las investigaciones infrinjan su cometido final, la de ayudar a otros, noveles y expertos, a criticar, analizar, incluso, a replicar la investigación en otros contextos. Es posible que detrás de estos resultados existan otras limitaciones, ${ }^{7}$ que ameritan realizar esfuerzos conjuntos entre investigadores, asesores y la institución propiamente dicha; algunos estudios apuntan que los universitarios tienen poco apoyo por parte del docente para publicar ${ }^{25}$ y muchos de ellos no conocen de procesos editoriales. ${ }^{26}$ Haciendo más difícil esta fase de la investigación.

Divulgación que está estrechamente relacionada con el impacto de una investigación, medida en función de las veces que un estudio es citado por otros investigadores, sea mediante artículo científico o mediante la tesis, para replicarlos o servir de base en otras investigaciones. ${ }^{27}$ En tal sentido, medir el impacto evidencia la contribución que el estudio hace a la sociedad, que busca la resolución de sus problemas; siendo que en este aspecto, los repositorios de tesis cumplen una labor fundamental en la difusión e impacto de las mismas; lo que exige al mismo tiempo, una mejora continua de la calidad de ellas, comprometiendo en este proceso de mejora tanto al estudiante como al docente y a los responsables del área de investigación, directores y decanos.

En el estudio, el porcentaje de tesis citadas como antecedentes en investigaciones a posteriori fue de $23 \%$ (gráfico 5), lo que significa una baja respuesta de las investigaciones a la comunidad científica nacional y regional, que ameritaría saber ¿por qué? Tal vez, la contestación estaría reflejada en la calidad científica y metodológica de las tesis en sí misma.

La Ley Universitaria ${ }^{12}$ refiere que es función obligatoria de la universidad producir conocimiento acorde con las necesidades de la sociedad, con especial énfasis en la realidad nacional. En la evaluación del impacto, las tesis publicadas como artículos científicos fueron las más citadas en otras tesis u otros artículos científicos; corroborando que la difusión facilita su captación por la comunidad académica científica. Por otro lado, la escasa repercusión de la tesis también puede ser reflejo de la poca respuesta que están dando a los problemas sociosanitarios del país.
Este dato muestra la importancia de que las tesis sean finalmente publicadas en artículos científicos, en revistas de impacto nacional o regional, resaltando que en esta fase el apoyo del docente asesor/investigador es prioritario y debe ser una práctica cada vez más frecuente en el ámbito universitario, ${ }^{5,28}$ dado la responsabilidad docente del asesor de enseñar cada vez más con mayor pericia y de aquello que hace cotidianamente. Paravic y Burgos ${ }^{7}$ afirman que los guías y asesores de tesis deben procurar la buena calidad de las tesis para producir manuscritos finales de calidad.

En tal sentido, la capacitación docente en investigación debe ser promovida y reforzada en las universidades, debido a su importancia en la formación de los profesionales y por qué nadie puede enseñar aquello que no practica. ${ }^{5,13}$ Cumpliendo así con la finalidad de la investigación, la de constituirse en medio para desarrollar y transformar el conocimiento en cualquier disciplina profesional.

Finalmente, la calidad de las tesis puede medirse de acuerdo a la dimensión que se estructure, determinando si las tesis fueron malas o buenas; además de recalcar la importancia de realizar este tipo de investigaciones para favorecer y objetivizar el crecimiento alcanzado respecto a la investigación en la universidad y en la profesión. En el estudio las tesis fueron de buena y regular calidad; siendo que el $40 \%$ cumplió apropiadamente el proceso de investigación y el rigor del método científico, con escasos estudios publicados en revistas científicas.

Entre algunas limitaciones que se pueden evidenciar en el estudio resaltan la pericia del investigador, quien siendo un novel cientista pudo haber sesgado la información en la fase de recolección. Acción que podrá corroborarse cuando el instrumento sea utilizado en otros estudios y ámbitos universitarios. Por otro lado, los resultados son válidos solo para la población analizada.

\section{Declaración de financiamiento y de conflictos de interés:}

El estudio fue financiado por el autor, declara no tener conflictos de interés.

\section{Contribución de auditoría:}

Guzmán DD: Concepción y diseño del estudio, recolección de datos, análisis e interpretación de los resultados y aprobación de la versión que será publicada 
Correspondencia:

Diego Armando Guzmán Díaz

Correo electrónico: dguzman@ correo.eeplt.edu.pe

\section{REFERENCIAS BIBLIOGRÁFICAS}

1. Mendoza P. Los grados académicos. Surgimiento y evolución. Anales de la facultad de medicina [internet]. 2000 [citado 22 jun 2015]; 61: 278-84. Disponible

en: http://sisbib.unmsm.edu.pe/bvrevistas/anales/v61_ n4/los_grad_acad.htm

2. Sistema Nacional de Evaluación, Acreditación y Certificación de la Calidad Educativa SINEACE. La educación superior en el Perú: Retos para el aseguramiento de la Calidad [internet]. 2013 [citado 22 jun 2015]. p.82. Disponible en: http://www.sineace.gob.pe/wp-

content/uploads/2013/08/Retos-para-el-

aseguramiento-de-la-calidad.pdf

3. Nicholson K. Quality assurance in higher education: a review of the literature [internet]. Nicholson K: s.n.: Jan 2011 [citado 22 de jun 2015]. [15 laudas]. Disponible en: http://cll.mcmaster.ca/COU/pdf/Quality\%20Assura nce\%20Literature\%20Review.pdf

4. Ley General de Educación. Ley 28044/2003 de 28 de julio. [internet]. Lima-Perú: Congreso de la República del Perú; 2003 [citado 22 jun 2015]. [cerca 36 laudas]. Disponible en: http://www.minedu.gob.pe/p/ley_general_de_educ acion_28044.pdf

5. United Nations Educational, Scientific and Cultural Organization UNESCO. Conferencia mundial sobre la educación superior - 2009: la nueva dinámica de la educación superior y la investigación para el cambio social y el desarrollo. [internet]. Paris-Francia: UNESCO; jul 2009 [citado 22 jun 2015]. p.09. Disponible en: http://www.unesco.org/education/WCHE2009/com unicado_es.pdf

6. Farci G. Patrones metodológicos en la evaluación de la productividad y producción investigativa. Investigación y Postgrado, Venezuela [serie de internet]. 2007 [citado 12 jun 2015]; 22(1): 187 205. Disponible en: http://www.redalyc.org/articulo.oa?id=65822108

7. Paravic KT, Burgos MM. Evaluación de calidad de resúmenes de tesis de un programa de magíster en enfermería. Cien enferm, Chile [serie de internet]. 2009 [citado 12 jun 2015]; 15(3): 55-68 Disponible en: http://www.scielo.cl/scielo.php?script=sci_arttext \&pid=S0717-95532009000300007
8. Merighi MAB, Gonçalves R, Ferreira FC. Estudio bibliométrico sobre disertaciones $\mathrm{y}$ tesis en enfermería con aproximación fenomenológica: Tendencias y perspectivas. Rev Latino-am Enfermagem, Brasil [serie de internet]. Jul-ago 2007 [citado 13 jun 2015]; 15(4): [07 laudas]. Disponible en: http://www.scielo.br/pdf/rlae/v15n4/es_v15n4a19. pdf

9. Mandujano-Romero E, Grajeda AP. Calidad de las tesis para obtener el título de médico cirujano, Universidad Nacional San Antonio Abad del Cusco - Perú, 2000-2009. Acta Med Per, Lima [serie de internet]. 2013 [citado 12 jun 2015]; 30(2): 70-74. Disponible en: http://www.scielo.org.pe/scielo.php?script=sci_artt ext\&pid=S1728-59172013000200004\&lang=es

10. Sanabria H, Bullón L. Exploración de la calidad de las tesis de Enfermería de la Facultad de Medicina de San Fernando. Universidades. 2000; 20 (1): 27 34.

11. Zavaleta RCJ. Calidad de la tesis de pregrado en una facultad de medicina [tesis de titulación]. [ internet]. Trujillo-Perú: Universidad Nacional de Trujillo; 2013 [citado 12 jun 2015]. Disponible en: http://dspace.unitru.edu.pe/xmlui/bitstream/handle/ $123456789 / 349 /$ ZavaletaReyes_C.pdf?sequence $=1$ \&isAllowed $=\mathrm{y}$

12. Ley universitaria. Ley $30220 / 2014$ de 8 de julio. El peruano, $\mathrm{n}^{\circ}$ 12914, (09-07-2003).

13. Ochoa-Vigo K, Ruiz-Garay M, Parodi-Carro A. Evaluación de tesis de pregrado en una escuela de enfermería: relato de experiencia Rev enferm Herediana, Lima. [serie de internet]. Ene-jun 2016 [citado 10 feb 2017]; 9(1): 62-67. Disponible en: http://dx.doi.org/10.20453/renh.v9i1.2865

14. Atuncar QFA, Gastulo MF. Autoevaluación para la acreditación en la Escuela Tezza: relato experiencia Cuid salud, Perú [serie de internet]. Jul-ago 2014. [citado 10 feb 2017]; 1(2): 101-112. Disponible en: http://eeplt.edu.pe/revista/index.php/Salud/article/v iew/26 
15. Universidad Ricardo Palma-Rectorado. Sistema de Calificación. [internet]. Lima-Perú: URP; 2016; [actualizado s.f.; citado 15 feb 2017]. Disponible en:

http://www.urp.edu.pe/relacionesuniversitarias/mo vilidad-estudiantil-estudiar-Universidad-sistemade-calificacion.html

16. Escuela de Enfermería Padre Luis TezzaPublicaciones Científicas. Cuidado y Salud/Kawsayninchis [internet]. Lima-Perú: EEPLT; 2014. [actualizado mar 2017; citado 3 de abr 2017]. Disponible en: http://www.eeplt.edu.pe/revista/

17. Mariner TA, Raile AM. Modelos y teorías en enfermería. $5^{\circ}$ ed. Madrid: Harcourt-Brace; 2005.

18. Organización de las Naciones Unidas-ONU. Centro de Noticias. Objetivos del Desarrollo Sostenible. [internet]. Ginebra: ONU; 2015 [actualizado s.f.; citado 22 mar 2017]. Disponible en:

http://www.un.org/sustainabledevelopment/es/heal th/

19. Juarez-Rolando P. Bibliometría para la evaluación de la actividad científica en ciencias de la salud. Revista enfermería herediana, Lima [serie de internet]. 2016 [citado 15 feb 2017] 9 (1): 57-61. Disponible

en: http://www.upch.edu.pe/vrinve/dugic/revistas/inde x.php/RENH/article/view/2864/2731

20. Universidad Politécnica Hispano Mexicana. Manual para la elaboración de tesis y trabajos de investigación. [internet] Puebla-México: UPHM; ago 2009 [citado 05 ene 2016]. p.91. Disponible en: http://www.uphm.edu.mx/manuales/Manualpara-elaboracion-de-tesis-y-trabajos-deinvestigacion.pdf

21. Pisconte SJ. Calidad de los trabajos de investigación para optar el título profesional de enfermería en la Universidad Nacional San Luis Gonzaga de Ica en el periodo 2008-2012. Revista enfermería a la vanguardia [serie de internet]. Enejun 2013 [citado 12 jun 2015]; 1(1): [cerca 07 laudas].

Disponible

en: http://www.unica.edu.pe/alavanguardia/index.php/ revan/article/view/2/2

22. Guzmán AM, Chanvergo VH, Guzman AB. Análisis científico y bibliométrico de las tesis de enfermería con la investigación científica Universidad Nacional Santiago Antúnez de Mayolo. Revista aporte santiaguino. 2009; 2(2): 313-319.

23. Burns N, Grove SK. Investigación en enfermería. 3 ed. España: Elservier; 2004.

24. Day R, Gastel B. Como escribir y publicar trabajos científicos. 4 ed. Washington: greenwordpress; 2008.

25. Pamo ROG. Estado actual de las publicaciones periódicas científicas medicas del Perú. Rev Med Hered [serie de internet]. 2005 [citado 20 oct 2009]. 16(1):65-73. Disponible en: http://www.scielo.org.pe/pdf/rmh/v16n1/v16n1ce1 .pdf

26. Mayta-Tristán P, Peña-Oscuvilca A. Importancia de la publicación en las sociedades científicas de medicina del Perú: estudio preliminar. CIMEL, Perú [serie de internet]. 2009 [citado 20 oct 2009]; 14(1):27-34. Disponible en: http://www.scielo.org.pe/pdf/cimel/v14n1/a05v14n $\underline{1 . p d f}$

27. Uribe ZJD, Cuadros MA. Las redes científicas en los grupos de la Universidad Pontificia Bolivariana sede-Medellín-Colombia. Revista Ciencias Estratégicas, Colombia [serie de internet]. Jul-dic 2011 [citado 15 feb 2017]; 19 (26): 212-225. Disponible en: https://revistas.upb.edu.co/index.php/cienciasestrat egicas/article/view/1092/1312

28. Sistema Nacional de Evaluación, Acreditación y Certificación de la Calidad Educativa-SINEACE. Modelo de acreditación para programas de estudio de educación superior universitaria [internet]. Lima-Perú: SINEACE; oct 2016. [citado 10 feb 2017]. [cerca 37 laudas]. Disponible en: https://www.sineace.gob.pe/wpcontent/uploads/2014/08/Anexo-1-nuevo-modeloprogramas-Resolucion-175.pdf 